\title{
Synthesis of Co, Ni and their Alloy Nanoparticles in Silica by Ion Implantation
}

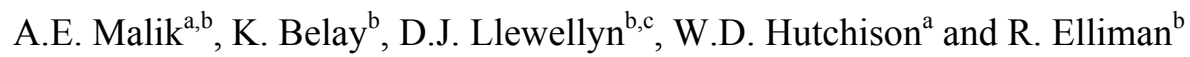 \\ ${ }^{a}$ School of Physical, Environmental and Mathematical Sciences, The University of New South Wales at \\ ADFA, Canberra ACT 2600. \\ ${ }^{\mathrm{b}}$ Electronic Materials Engineering Department, Research School of Physics and Engineering, Australian \\ National University, Canberra, ACT 0200, Australia. \\ ${ }^{\mathrm{c}}$ Centre for Advanced Microscopy, Australian National University, Canberra, ACT 0200, Australia.
}

Magnetic nano-particles embedded in semiconductor and insulating matrices are of interest in the study of fundamental properties such as superparamagnetism, magneto-resistivity and magneto-optics, as well as for technological applications like data storage and spintronics [1-4]. The use of ion beam techniques, easily adapted to patterning, as part of the fabrication process in such cases is clearly a sensible approach. Ion implantation of metallic ions into an insulating matrix is a powerful technique for the elaboration of nanosized metal particles. These nanosized particles are characterized by novel properties that are significantly different from those of the corresponding bulk phase [1].

Nanoparticles of metallic Nickel, Cobalt and a Co-Ni alloy have been synthesized, via ion implantation and thermal annealing, within $100 \mathrm{~nm}$ silica thin films thermally grown on silicon substrates. The starting substrate consisted of a $100 \mathrm{~nm} \mathrm{SiO} 2$ layer thermally grown on (100) oriented $\mathrm{Si}$ wafers. Separate regions of the silica layer were implanted with $50 \mathrm{keV} \mathrm{Ni}$ and Co ions with fluences of $6 \times 10^{16}$ ions $/ \mathrm{cm}^{2}$, or for the Co-Ni alloy, sequentially implanted with $\mathrm{Co}$ and then $\mathrm{Ni}$ ions each with a fluence of $3 \times 10^{16}$ ions $/ \mathrm{cm}^{2}$. According to the Monte-Carlo ion-range simulation code SRIM 2007 [5], the average range of both Ni and $\mathrm{Co}$ ions in $\mathrm{SiO}_{2}$ is $\sim 43 \mathrm{~nm}$ for the above mentioned energies.

After implantation the samples were annealed at $900^{\circ} \mathrm{C}$ in a nitrogen atmosphere for one hour. The size and spatial distributions of the nanoparticles were measured by using Cross-Section Transmission Electron Microscopy (XTEM) of the samples. The crystal structure of the nanoparticles was determined by using glancing angle X-ray diffraction measurements (XRD).

Fig.1. shows bright-field, XTEM images of samples implanted with (a) Ni, (b) Co and (c) Co-Ni alloy after one hour annealing in $\mathrm{N} 2$ at $900^{\circ} \mathrm{C}$. In all the cases investigated, well defined spherical particles can be observed. It is evident from the images that size and size distributions are very different in the three cases, depending explicitly on the implanted species. Both Ni and Co-Ni alloy samples showed lesser diffusion in silica as compared to Co sample. Long range diffusion of the Co was also observed in these samples giving rise to nanocrystals in the vicinity of the $\mathrm{SiO}_{2}$ - $\mathrm{Si}$ interface. 
Fig.2. shows the results obtained after the samples were examined using (XRD). Fig.2.(a) shows the Ni pattern has peaks consistent with the presence of a cubic phase with all prominent peaks at $44.5^{\circ}, 76.5^{\circ}$ and $93.1^{\circ}$ being present. Fig.2.(b) shows that the Co is present in two different phases. Peaks at $44.2^{\circ}$ and a relatively smaller peak at $47.5^{\circ}$ are signature peaks for fcc (111) and hcp (101) phases of Co. The peaks between $50^{\circ}$ and $57^{\circ}$ degrees are due to the substrate and are present in all the patterns. Fig.2.(c) shows the Co$\mathrm{Ni}$ alloy sample where the pattern is consistent with fcc structure. The peaks present at $41.5^{\circ}, 44.4^{\circ}, 51.72^{\circ}, 60.8^{\circ} 76.3^{\circ}$ arise from the (111), (200), (220) planes of the fcc Co-Ni alloy phase.

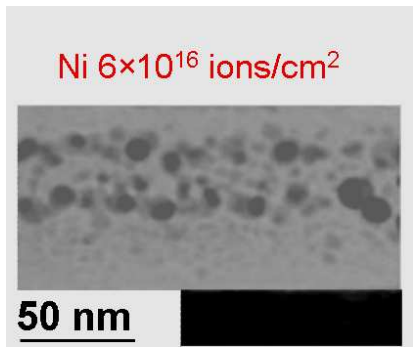

(a)

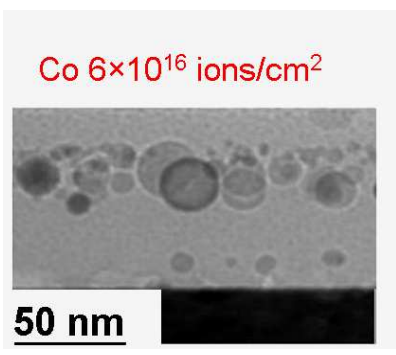

(b)
Co $3 \times 10^{16} \mathrm{ions} / \mathrm{cm}^{2}$ $\mathrm{Ni} 3 \times 10^{16} \mathrm{ions} / \mathrm{cm}^{2}$

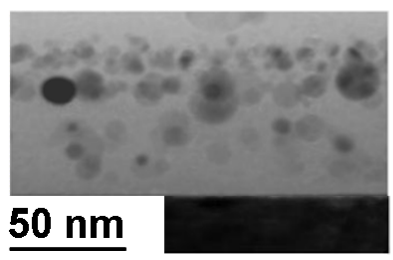

(c)

Fig.1. XTEM bright-field images of (a) Ni, (b) Co and (c) Co-Ni alloy nanoparticles formed after annealing.

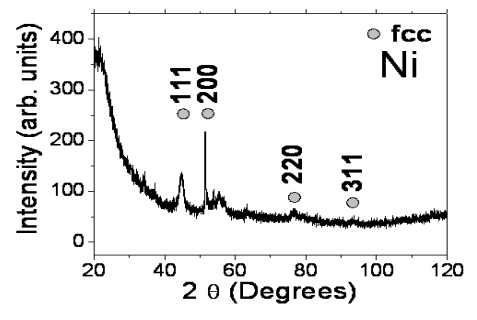

(a)

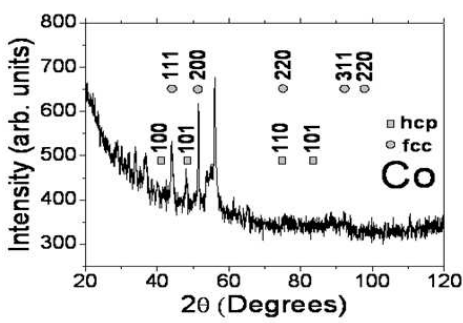

(b)

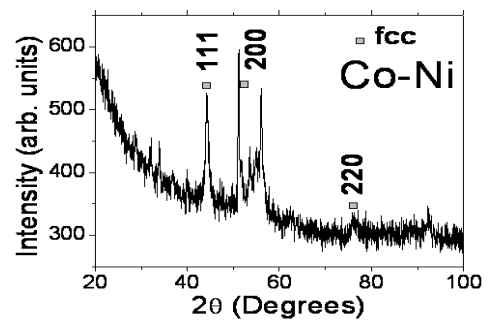

(c)

Fig. 2. XRD patterns of (a) Ni, (b) Co and (c) Co-Ni nanoparticles fabricated by implantation and annealing.

\section{References}

[1] Meldrum A, Richard F H Lynn A B and White C W (2001) Adv. Mater. 131431.

[2] Mattei G, de Julian C F, Mazzoldi P and Sada C (2002) Chem. Mater. 143440.

[3] Xian-Ming L, Shao-Yun F and Chuan-Jun H (2004) J. Mag. Mat. 281234.

[4] Cintora-Gonzaler O, Muller D, Estournes C, Richard-Plouet M, Poinsot R, Grob J J and Guille J (2001) Nucl. Inst. Methods Phys. B. 178144.

[5] Ziegler F, Biersack J P, Littmark U (1985) The Stopping and Range of Ions in Solids, (New York : Pergamon) http://www.srim.org/. 